\title{
Functional Coupling Between Substantia Nigra and Basal Ganglia Homologues in Amphibians
}

\author{
Kim L. Hoke \\ University of Texas at Austin
}

\author{
Michael J. Ryan \\ University of Texas at Austin and Smithsonian Tropical \\ Research Institute
}

\author{
Walter Wilczynski \\ Georgia State University
}

\begin{abstract}
Neuroanatomical and pharmacological experiments support the existence of a homologue of the mammalian substantia nigra-basal ganglia circuit in the amphibian brain. Demarcation of borders between the striatum and pallidum in frogs, however, has been contentious, and direct evidence of functional coupling between the putative nigral and striatal homologues is lacking. To clarify basal ganglia function in anurans, the authors used expression of immediate-early gene egr-1 as a marker of neural activation in the basal ganglia of túngara frogs (Physalaemus pustulosus). Regional variation in egr-1 mRNA levels distinguished striatal and pallidal portions of the basal ganglia and supported the grouping of the striatopallidal transition zone with the dorsal pallidum. As further evidence for a functional coupling between the dopaminergic cells in the posterior tuberculum (the putative substantia nigra homologue) and the basal ganglia, a positive relationship was demonstrated between the size of the dopaminergic cell population and the neural activation levels within the dorsal pallidum.
\end{abstract}

Keywords: anuran, basal ganglia, tyrosine hydroxylase, immediate-early gene expression, egr-1

Previous research has proposed that homologous basal ganglia circuits may exist in both amphibians and mammals (reviewed in Endepols, Schul, Gerhardt, \& Walkowiak, 2004; Marín, Smeets, \& González, 1998). The "direct" basal ganglia pathway in mammals involves a dopaminergic projection from the substantia nigra pars compacta $(\mathrm{SNc})$ to the striatum. The output of the striatum inhibits activity in the pallidum and the substantia nigra pars reticulata $(\mathrm{SNr})$, and output pathways from the pallidum and the $\mathrm{SNr}$ reach motor centers in the brainstem. Although details vary, this basic circuit appears to be an ancestral trait in tetrapods (Marín et al., 1998).

In amphibians, the proposed homologous circuit to the mammalian nigrostriatal pathway includes the posterior tuberculum (PT), striatum, and dorsal pallidum. Catecholaminergic cells in the PT contain dopamine (González \& Smeets, 1991) and project to the striatum (Marín, González, \& Smeets, 1997b; Marín, Smeets, \& González, 1997). On the basis of developmental analyses, these cells are proposed to be homologous to the dopaminergic SNc neurons in mammals (reviewed in Marín et al., 1998; Smeets \&

Kim L. Hoke, Section of Integrative Biology, University of Texas at Austin; Michael J. Ryan, Section of Integrative Biology, University of Texas at Austin, and Smithsonian Tropical Research Institute, Balboa, Panama; Walter Wilczynski, Department of Psychology and Center for Behavioral Neuroscience, Georgia State University.

We are grateful to the National Science Foundation (Grant NSF IBN 9816564) and the National Institute of Mental Health (Grant NIH MH 057066) for funding.

Correspondence concerning this article should be addressed to Kim L. Hoke, Section of Integrative Biology, University of Texas at Austin, 1 University Station C0930, Austin, TX 78712. E-mail: khoke@psych.stanford.edu
González, 2000). Chemoarchitecture and anatomical connections suggest that the amphibian basal ganglia consist of the homologues of the mammalian striatum (rostrally) and dorsal pallidum (caudally), with an intermediate portion that shares some characteristics with the striatum and other characteristics with the pallidum (Endepols, Schul, et al., 2004; Mülhenbrock-Lenter, Endepols, Roth, \& Walkowiak, 2005; see also Marín, González, \& Smeets, 1997c). Major outputs of the basal ganglia include direct and indirect paths to motor areas of the brainstem (Marín, González, \& Smeets, 1997a; Wilczynski \& Northcutt, 1983b).

Functional similarities between mammalian and amphibian basal ganglia circuits exist as well. The dopaminergic projection to the striatum has been implicated in the regulation of motor output in amphibians by studies that examined the behavioral effects of either basal ganglia lesions or pharmacological manipulation of dopaminergic transmission. Telencephalic lesions delay or abolish visual orientation toward contralateral prey stimuli if those lesions damage the striatum and amygdala, although which of these structures was critical could not be distinguished (Patton \& Grobstein, 1998). Specific striatal lesions abolish prey-catching behavior in toads (Finkenstädt, 1989). In addition, striatal lesions abolish phonotactic responses to mating calls in females (Walkowiak, Berlinger, Schul, \& Gerhardt, 1999).

Pharmacological manipulations alter motor behaviors in several contexts. Treatment with the neurotoxin MPTP induces rigidity and tremor, among other generalized motor deficits, in amphibians (Barbeau, Dallaire, Buu, Poirier, \& Rucinska, 1985; Barbeau, Dallaire, Buu, Veilleux, et al., 1985). Treatment with the nonspecific dopamine agonist apomorphine decreases prey-oriented locomotion and increases snapping at food in toads (Glagow \& 
Ewert, 1996, 1997, 1999) and decreases climbing behavior while preserving swimming in a frog (Chu \& Wilczynski, 2007). One study specifically implicated the PT in dopaminergic effects on motor behaviors: Lesioning of dopaminergic cells with 6-hydroxydopamine disrupts reproductive phonotaxis behaviors to an extent that correlates with the number of remaining dopaminergic cells in the PT (Endepols, Roden, Luksch, Dicke, \& Walkowiak, 2004). This last study suggested a strong functional coupling between these dopaminergic cells and the striatum in amphibians, similar to that suggested to be the case for the mammalian nigrostriatal system. In mammals, increased dopamine activity enhances striatal function, whereas disorders such as Parkinson's disease, which are caused by decreased dopaminergic innervation of the basal ganglia, include a decrease in striatal function (reviewed in Nicola, Surmeier, \& Malenka, 2000).

A strong functional coupling between nigral dopaminergic and striatal areas suggests that individual or group differences in the size of dopaminergic populations would correlate with activity differences in the basal ganglia. One experimental study (Glagow \& Ewert, 1999) examined glucose utilization in the basal ganglia following pharmacological manipulation with apomorphine and demonstrated decreased striatal 2-deoxyglucose uptake, accompanied by a decrease in prey-oriented behavior, following apomorphine treatment. The global application of the dopamine agonist and the widespread changes in neural activation patterns, however, prevented the strong inference that dopamine from the PT in particular regulates basal ganglia activity. In this study, we adopted a different approach, in that we compared natural variation in the number of immunoreactive PT dopaminergic cells with levels of immediate-early gene activation to determine whether increased dopamine levels in the PT correlate with higher functional activity in the basal ganglia.

In the present study, we examined both regional variation in functional activation within the basal ganglia and functional relationships between dopaminergic cells in the PT and basal ganglia activation. We used immediate-early gene $e g r-1$ mRNA levels as a marker for neural activation, as in previous studies (Hoke et al., 2004; Hoke, Ryan, \& Wilczynski, 2005, 2007), and used immunohistochemistry for tyrosine hydroxylase (TH) protein as a marker for dopaminergic PT neurons. TH is an enzyme in the synthetic pathway shared by the catecholamines dopamine and norepinephrine. The TH-positive cell population in the PT has been shown to be dopaminergic in some amphibians (González \& Smeets, 1991). First, we assessed regional variation in egr-1 mRNA abundance as a means of distinguishing functional domains within the basal ganglia of frogs to argue whether the intermediate basal ganglia is functionally related to the striatum rostrally or to the pallidum caudally. To describe the functional coupling of the PT and the basal ganglia, we next tested the correlation between the density of TH-immunoreactive neurons in the PT and egr-1 mRNA levels in the striatum and pallidum of female túngara frogs (Physalaemus pustulosus).

\section{Method}

\section{Subjects}

To assess regional differences in neural activation of the basal ganglia, we analyzed brains of male túngara frogs used in two experiments published previously (Hoke et al., 2004; Hoke, Ryan, $\&$ Wilczynski, 2007). Briefly, calling males ( $n \mathrm{~s}=33$ and 39, respectively, from the two experiments for frogs with all eight regional measures) were collected at natural breeding aggregations near the Smithsonian Tropical Research Institute in Gamboa, Panama $\left(9^{\circ} 07.0^{\prime} \mathrm{N}, 79^{\circ} 41.9^{\prime} \mathrm{W}\right)$, and were transported to the laboratory. Frogs were kept in sound attenuation chambers for $2 \mathrm{hr}$ prior to experiments and then were treated in one of the following stimulus conditions: silence, conspecific advertisement calls (whines or whine-chucks), heterospecific advertisement calls ( $P$. enesefae whines), conspecific aggressive calls (mews), or control stimuli (conspecific chuck excised from natural whine-chucks). Stimuli were broadcast at $82 \mathrm{~dB}$ SPL (ref. $20 \mu \mathrm{Pa}$ ) for $15 \mathrm{~min}$ (Hoke et al., 2004) or 30 min (Hoke et al., 2007).

To measure coupling between basal ganglia functional activation and the dopaminergic system, we collected female $P$. pustulosus between $2000 \mathrm{~h}$ and $2200 \mathrm{~h}$ from 2 July to 11 July 2005 Autoridad Nacional del Ambiente del República de Panamá approved the collection, animal protocols, and exportation of tissue. Amplexed pairs of frogs were found at breeding ponds near Gamboa, Panama, and were brought to the laboratory. Females were placed in moist plastic bags inside a sound isolation chamber that measured approximately $40-50 \mathrm{~cm}$ in each dimension. After 2-2.5 hours without stimulation, the frogs were exposed to a natural heterospecific $P$. petersi call ( $n=3$, with successful image processing for both $e g r-1$ and $\mathrm{TH}$ ) or to a natural $P$. pustulosus whine $(n=8)$. Acoustic stimuli were broadcast at $72 \mathrm{~dB}$ SPL (ref $20 \mu \mathrm{Pa}$ ) from an amplified speaker (Radio Shack, Forth Worth, $\mathrm{TX})$ at a rate of 1 call per $2 \mathrm{~s}$ for $15 \mathrm{~min}$, and then frogs were kept in silence for $15 \mathrm{~min}$ more.

\section{Tissue Processing}

Following the stimulus exposure, we rapidly decapitated the frogs and immediately froze their brains in liquid nitrogen for subsequent cryostat sectioning. We processed brains of males for radioactive in situ hybridization to localize egr- $1 \mathrm{mRNA}$, as previously described (Hoke et al., 2004; Hoke et al., 2007). We processed alternate sections of female brains, either for radioactive in situ hybridization against $e g r-1$ or for TH immunocytochemistry, as follows. Brain sections were fixed in $4 \%$ paraformaldehyde for $10 \mathrm{~min}$ and then were treated with $0.1 \%$ sodium borohydrate in phosphate buffered saline (PBS) followed by $1 \%$ hydrogen peroxide in PBS for 15 min each. Nonspecific antibody binding was blocked with $2 \%$ normal horse serum and free avidin (Vector Laboratories, West Grove, PA), and then monoclonal mouse anti-TH antibody (Chemicon International, Temecula, CA) was applied overnight in $2 \%$ serum and free biotin (Vector Laboratories). TH immunoreactivity was visualized with sheep anti-mouse conjugated to fluorescein isothiocyanate (Chemicon International), and cells were counterstained with propidium iodide.

\section{Image Analysis}

We estimated average egr-1 mRNA levels in the basal ganglia as previously described (Hoke et al., 2004; Hoke et al., 2007). Briefly, we analyzed 1,000 photomicrographs using a customized automated counting procedure to calculate the number of silver grains divided by area covered by cells in a standard-size sampling 
frame. Sampling procedures differed for male and for female frogs. For each male, we measured silver grain density in four sections through the basal ganglia spaced by at least $32 \mu \mathrm{m}$, sampling one dorsal and one ventral photomicrograph in each of the four sections as described (Hoke et al., 2007). The two rostral sections corresponded to striatum as identified by Endepols, Schul, et al. (2004), and the two caudal sections spanned the striatopallidal transition area (Endepols, Schul, et al., 2004). For the females, we carried out more extensive sampling throughout the basal ganglia. We captured one dorsal and one ventral photomicrograph in every section that contained basal ganglia (all separated by at least $32 \mu \mathrm{m}$, due to preparation of three sets of alternate sections for each brain). We grouped the sections into rostral, intermediate, and caudal thirds. The caudal third encompassed sections in which amygdala was present medially, and the intermediate third was typically marked by more pronounced lamination in the lateral septum and by narrower width of the basal ganglia (see Figure 1).

To count TH-positive cells, we used confocal microscopy to visualize TH immunolabeling in all sections containing the PT. Because of possible rostrocaudal variation in $\mathrm{TH}-$ immunoreactive cells, we selected only frogs in which the PT was clearly visible in all sections through the caudal hypothalamus; frogs in which the PT was indistinct, torn, or too angled for clear determination of PT boundaries were excluded. We collected stacks of 1- $\mu \mathrm{m}$ images through each caudal hypothalamic section using Leica SP2AOBS (Leica Microsystems, Bannockburn, IL) and then collapsed stacks into a summary image for counting cells with Leica Confocal Software (Leica Microsystems, Bannockburn, IL; see Figure 2). Note that sections were spaced by $32 \mu \mathrm{m}$, so cells were not counted twice in adjacent sections. We counted every $\mathrm{TH}$-immunoreactive cell within the borders of the PT in 4-12 sections for each individual and reported the average TH-positive cell number per tissue section imaged (TH-immunoreactive cell density).

\section{Statistics}

We used SPSS Version 11 for statistical analyses. To determine regional variation in $e g r-1$ mRNA levels in the basal ganglia, we used a repeated-measures analysis of variance (ANOVA) with the eight egr-1 measures from each male subject as the dependent variables. Dorsal or ventral position was nested within the four levels of rostrocaudal position. We combined information from the two separate experiments with male $P$. pustulosus by normalizing the results for each experiment separately (dividing each measure of silver grain density by the overall average of basal ganglia egr-1 expression in all subjects in that experiment) and by including experiment as a between-subjects factor. We then assessed withinsubject effects of rostrocaudal position, dorsoventral position, Rostrocaudal $\times$ Dorsoventral interaction, and the interaction of each of those with experiment number. We used pairwise comparisons of estimated marginal means with Bonferroni correction as post hoc analyses to determine which levels of factors were significantly different.

We further analyzed regional variation using principalcomponents analysis. Because experiment number had no significant main or interaction effects in the ANOVA analysis, we combined results from the two experiments and used principalcomponents analysis to group the eight egr-1 measures by similarity. We used promax rotation with Kaiser normalization $(\kappa=4)$ and assessed the component loadings in the pattern matrix to argue for functional groupings of the eight regional measures into distinct striatal and pallidal divisions.

For the experiments with female $P$. pustulosus that addressed coupling of the basal ganglia and dopaminergic inputs from the PT, we first confirmed a similar regional pattern of activation using a repeated-measures ANOVA. We calculated six average egr-1 levels that represented the activation in the dorsal and ventral portions of the rostral, intermediate, and caudal basal ganglia (averaging silver grain densities from 1 to 4 sections for each

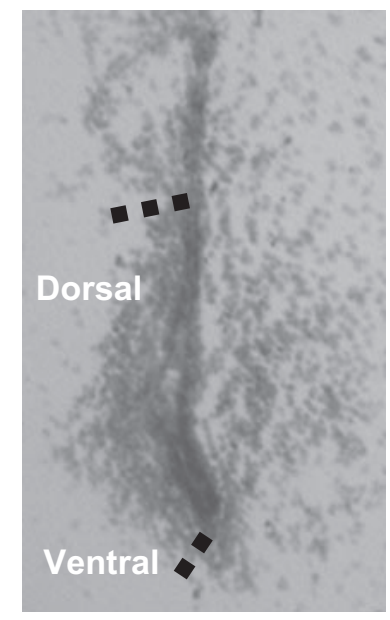

Rostral

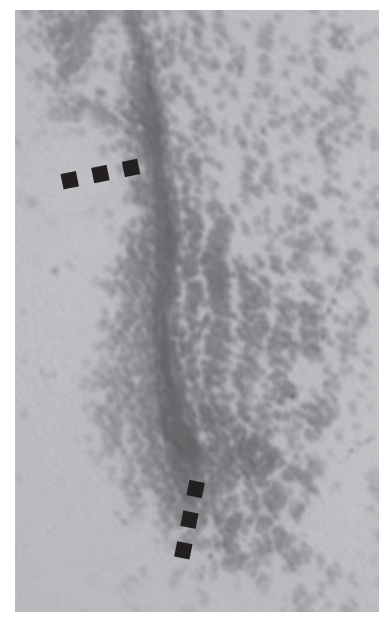

Intermediate

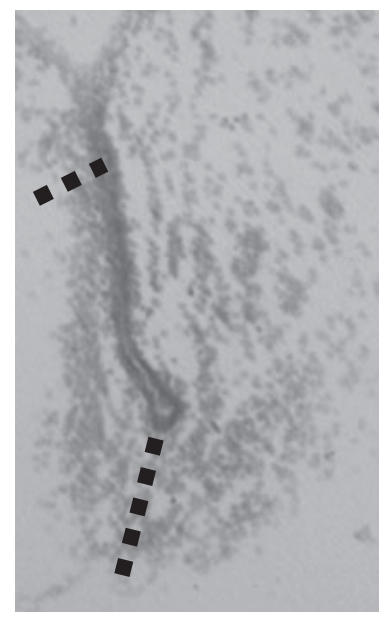

Caudal

Figure 1. Anatomical boundaries of striatal, intermediate, and pallidal portions of the basal ganglia in $P$. pustulosus brains. Nissl-stained transverse sections through brains of female $P$. pustulosus show typical sections in the rostral/striatal, intermediate/striatopallidal transition, and caudal/pallidal portions of the basal ganglia. Dashed lines mark approximate dorsal and ventral borders of basal ganglia, with medial border at the ventricle. 

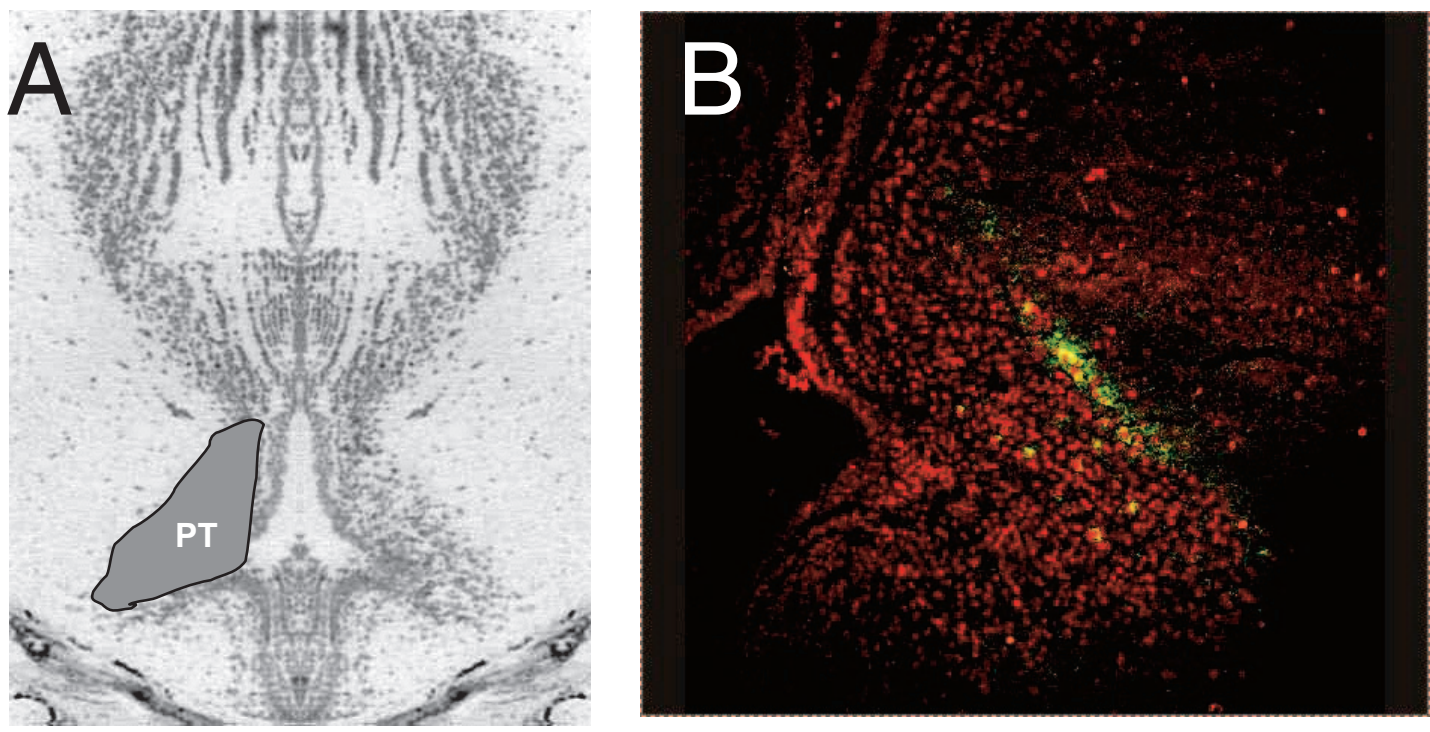

Figure 2. Photomicrographs showing tyrosine hydroxylase (TH) immunocytochemistry in the posterior tuberculum (PT). A: Image of Nissl-stained transverse section indicating the PT. The right half of the image is the mirror image of the left half, for clarity. B: Confocal image showing TH-immunoreactive cells (green) in the PT in a transverse section. Cell nuclei are counterstained with propidium iodide (red) for visualization of brain region boundaries.

positional measure). We then used a repeated-measures ANOVA to determine rostrocaudal and dorsoventral variation and used pairwise comparisons of estimated marginal means to determine which levels of the factor differed significantly. On the basis of this analysis (detailed below in the Results section), we obtained averaged estimates of striatal and pallidal egr-1 levels: Egr-1 mRNA abundance in the striatum was calculated by averaging dorsal and ventral measures in the rostral basal ganglia, whereas egr-1 expression in the pallidum was represented by the average silver grain densities from the dorsal and ventral portions of the intermediate and caudal basal ganglia. We then used the Pearson correlation coefficient between striatal or pallidal egr-1 levels and TH-positive cell number in the PT to address functional coupling between these systems.

\section{Results}

\section{Regional Variation in Basal Ganglia Activation}

We determined the rostrocaudal and dorsoventral variation in egr-1 expression across the extent of the rostral and intermediate basal ganglia in two sets of brains from male P. pustulosus. We found significant rostrocaudal variation across the four sections selected for analysis in each frog, $F(3,210)=16.9, p<.001$, as well as a Rostrocaudal $\times$ Dorsoventral interaction effect, $F(3$, $210)=4.596, p=.004$. Egr-1 mRNA abundance did not vary consistently with dorsoventral position, nor were there main or interaction effects that distinguished the two experiments (see Table 1). Post hoc comparisons of estimated marginal means showed that egr-l expression in the two rostral positions was significantly different from expression in the caudal half of the basal ganglia to account for the main rostrocaudal effect (see Figure 3 and Table 2). The Rostrocaudal $\times$ Dorsoventral interac- tion effect arose because the ventral portion of the secondmost rostral section was more similar to its caudal neighbor than to its rostral neighbor, in contrast to the opposite finding in the dorsal position (see Table 3). Thus, we concluded that the intermediate portion of the $P$. pustulosus basal ganglia (pallidal/striatal transition zone) is functionally distinct from the rostral portion (striatum proper, based on Endepols, Schul, et al., 2004).

We used principal-components analysis as a second statistical approach to analyze regional variation in functional activation of the basal ganglia. As we had found no differences based on experiment in the repeated-measures ANOVA analysis discussed above, we combined $e g r-1$ mRNA measures from the two experiments in a principal-components analysis. We extracted two factors with eigenvalues over 1.0 that explained $62 \%$ of the total variance in the eight $e g r-1$ density measures. The rotated solution showed clear separation of the dorsal and ventral egr-l levels in

Table 1

Within-Subjects Effects of Rostrocaudal $(R C)$ and Dorsoventral (DV) Position on egr-1 Levels in the Basal Ganglia in Two Experiments (EXPT)

\begin{tabular}{lrrlrr}
\hline \multicolumn{1}{c}{ Effect } & \multicolumn{1}{c}{$S S$} & $d f$ & \multicolumn{1}{c}{$M S$} & \multicolumn{1}{c}{$F$} & \multicolumn{1}{c}{$p$} \\
\hline RC & 17.747 & 3 & 5.916 & 16.922 & $<.001$ \\
RC $\times$ EXPT & 0.412 & 3 & 0.137 & 0.392 & .759 \\
Error $($ RC) & 73.41 & 210 & 0.35 & & \\
DV & 0.106 & 1 & 0.106 & 0.516 & .475 \\
DV $\times$ EXPT & 0.472 & 1 & 0.472 & 2.293 & .134 \\
Error $($ DV) & 14.419 & 70 & 0.206 & & \\
RC $\times$ DV & 1.876 & 3 & 0.625 & 4.596 & .004 \\
RC $\times$ DV $\times$ EXPT & 0.831 & 3 & 0.277 & 2.035 & .11 \\
Error $($ RC $\times$ DV) & 28.581 & 210 & 0.136 & & \\
\hline
\end{tabular}


the two rostral sections from the egr-1 levels in the two caudal sections on the basis of the pattern matrix (see Table 4; bold type indicates strong loadings on a factor). The two components were positively correlated $(r=.442)$. With the repeated-measures ANOVA results, we thus have two lines of evidence that the intermediate basal ganglia functions separately from the rostral portion.

We confirmed and extended the regional variation in basal ganglia activation in the smaller data set from female brains. These brains had both dorsal and ventral measurements of egr-1 from every section that contained basal ganglia, divided into rostral, intermediate, and caudal portions. A repeated-measures ANOVA that was based on average dorsal and ventral egr-1 mRNA levels in sections in each rostrocaudal third showed significant withinsubjects variation based on rostrocaudal position, $F(2,20)=$ $11.229, p=.001$, but not on dorsoventral position, $F(1,10)=$
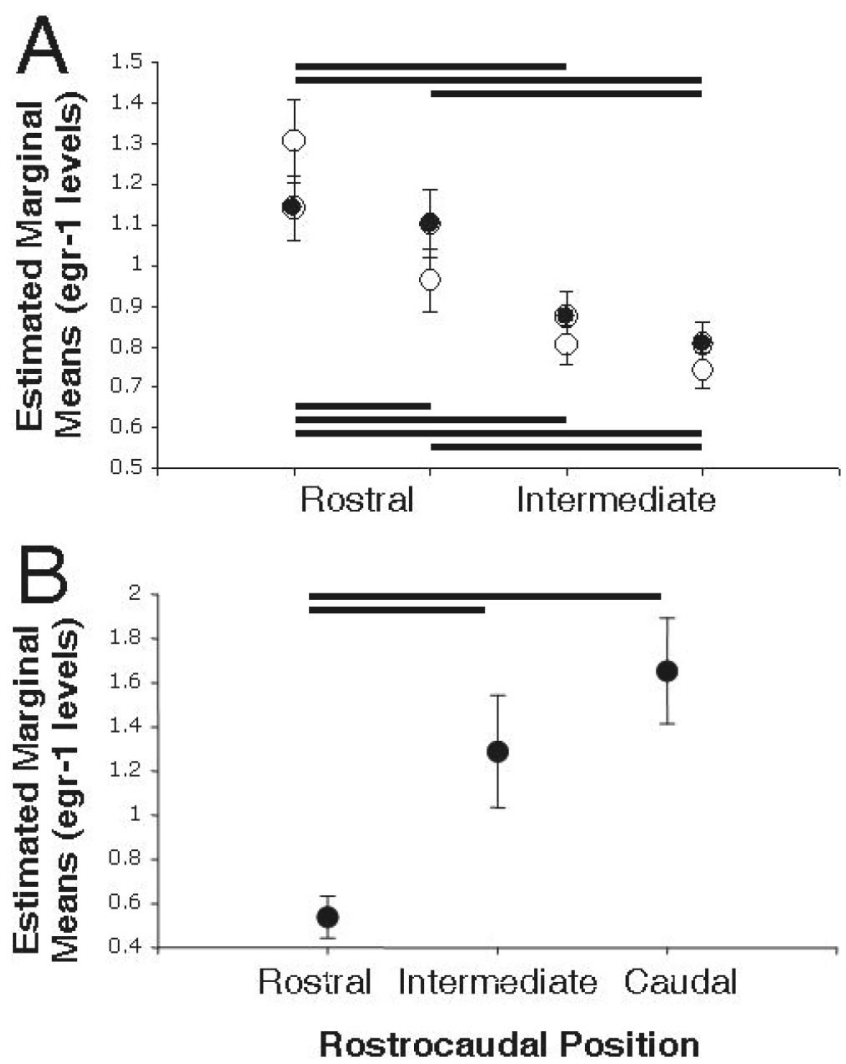

Figure 3. Regional variation in egr-1 levels in the basal ganglia along rostrocaudal and dorsoventral axes. A: Estimated marginal means for relative $e g r-1$ levels at each rostrocaudal position in the ventral (open circles) and dorsal (closed circles) basal ganglia of male P. pustulosus. Error bars indicate standard errors of the mean, and horizontal bars mark post hoc pairwise comparisons significant at the $p=.05$ level, with dorsal and ventral differences depicted above and below the graph, respectively. B: Estimated marginal means for egr-1 levels in the rostral, intermediate, and caudal basal ganglia of female $P$. pustulosus. Note that in contrast to Panel A, the rostral basal ganglia has lower activation than do intermediate/ caudal portions. This finding may indicate a sex difference or a difference based on acoustic stimuli used in the experiments. Error bars indicate standard errors of the mean, and horizontal bars mark post hoc pairwise comparisons significant at the $p=.05$ level.
Table 2

Pairwise Comparisons of egr-1 mRNA Levels in Each Rostrocaudal Position ( 1 = Rostralmost $)$ of the Basal Ganglia

\begin{tabular}{ccccr}
\hline $\begin{array}{c}\text { Rostrocaudal } \\
\text { Site } 1\end{array}$ & $\begin{array}{c}\text { Rostrocaudal } \\
\text { Site 2 }\end{array}$ & $\begin{array}{c}\text { Mean } \\
\text { difference }\end{array}$ & SE & \multicolumn{1}{c}{$p$} \\
\hline 1 & 2 & 0.191 & 0.076 & .083 \\
& 3 & 0.385 & 0.071 & $<.001$ \\
& 4 & 0.45 & 0.079 & $<.001$ \\
2 & 3 & 0.194 & 0.072 & .052 \\
& 4 & 0.259 & 0.073 & .004 \\
3 & 4 & 0.0648 & 0.042 & .745 \\
\hline
\end{tabular}

$0.608, p=.454$, or on Rostrocaudal $\times$ Dorsoventral interaction, $F(2,20)=1.588, p=.229$. Post hoc pairwise comparisons of estimated marginal means found significant differences between the rostral-most position and both intermediate and caudal sites ( $p=.016$ and $p=.003$, respectively, Bonferroni corrected) but no difference between intermediate and caudal sites $(p=.601)$. Thus, in accordance with the above results from males, the intermediate basal ganglia is functionally related to the pallidum and not to the striatum.

\section{Functional Coupling of Pallidal and Dopaminergic Systems}

We measured relative striatal and pallidal egr- 1 mRNA levels and $\mathrm{TH}$-immunoreactive cell density in 3 frogs exposed to heterospecific calls and in 8 frogs that heard conspecific calls. We found that TH-positive cell density was significantly correlated with egr-1 mRNA abundance in the pallidum $(r=.666, p=.025)$ but not in the striatum $(r=.263, p=.434)$. Although sample sizes were small, there was no suggestion of a difference between individuals based on acoustic treatment (see Figure 4). We conclude that dopaminergic cells in the PT are functionally related to basal ganglia activation.

\section{Discussion}

We have presented two lines of evidence to clarify the shared structure and function of the basal ganglia across amphibians and mammals. We showed that putative striatal and pallidal homologues have distinct patterns of functional activation and argued that the striatopallidal transition zone identified by Endepols, Schul, et al. (2004) is functionally related to the pallidum. We furthermore demonstrated a functional relationship between neural activation in the basal ganglia and dopaminergic projections from the PT, which further bolsters the hypothesis that homologous basal ganglia circuits regulate motor behaviors in tetrapods.

Previous researchers have used regional variation in anatomical connectivity and neurochemical markers to propose amphibian homologues of the striatum and dorsal pallidum, with a transition zone sharing some characteristics of striatal and pallidal regions (Endepols, Schul, et al., 2004; Mülhenbrock-Lenter et al., 2005). We argue, on the basis of neural activation patterns, that the transition zone is functionally related to the caudal/pallidal rather than to the rostral/striatal portion of the basal ganglia. Three separate experiments show significant differences between the egr-1 expression levels in the rostral and intermediate thirds of the 
Table 3

Pairwise Comparisons of egr-1 mRNA Levels in Each Rostrocaudal Position Separated Into

Dorsal and Ventral Halves of the Basal Ganglia

\begin{tabular}{|c|c|c|c|c|c|}
\hline $\begin{array}{l}\text { Dorsoventral } \\
\text { position }\end{array}$ & $\begin{array}{l}\text { Rostrocaudal } \\
\text { Site } 1\end{array}$ & $\begin{array}{l}\text { Rostrocaudal } \\
\quad \text { Site } 2\end{array}$ & $\begin{array}{c}\text { Mean } \\
\text { difference }\end{array}$ & $S E$ & $p$ \\
\hline \multirow[t]{6}{*}{ Dorsal } & 1 & 2 & 0.0395 & 0.082 & 1 \\
\hline & & 3 & 0.268 & 0.077 & .006 \\
\hline & & 4 & 0.335 & 0.081 & .001 \\
\hline & 2 & 3 & 0.228 & 0.085 & .054 \\
\hline & & 4 & 0.295 & 0.081 & .003 \\
\hline & 3 & 4 & 0.0669 & 0.055 & 1 \\
\hline \multirow[t]{6}{*}{ Ventral } & 1 & 2 & 0.342 & 0.099 & .006 \\
\hline & & 3 & 0.503 & 0.099 & $<.001$ \\
\hline & & 4 & 0.565 & 0.105 & $<.001$ \\
\hline & 2 & 3 & 0.161 & 0.077 & .241 \\
\hline & & 4 & 0.223 & 0.081 & .045 \\
\hline & 3 & 4 & 0.0628 & 0.048 & 1 \\
\hline
\end{tabular}

basal ganglia. None of these experiments show consistent patterns of dorsoventral variation that would support a primary division of the basal ganglia into dorsal and ventral striatum, as has been proposed (Wilczynski \& Northcutt, 1983a). Although we find some evidence of a rostrocaudal difference within the intermediate striatum (see Table 3), the combined analyses of repeatedmeasures ANOVA and of principal components suggest that the intermediate portion of the basal ganglia is primarily pallidal in function. Additional analyses of rostral, intermediate, and caudal egr-1 expression in both male and female amphibians that describe how egr-1 levels in each region vary with motor behaviors in different contexts should add information to clarify the functional roles of the striatal and pallidal divisions of the basal ganglia in anurans.

The finding of a positive correlation between density of THimmunoreactive cells in the PT and a measure of neural activation in the dorsal pallidum provides new evidence that supports the existence of a functionally homologous $\mathrm{SNc}$-basal ganglia circuit in tetrapods. The density of TH-immunoreactive cells in the PT is a function both of the total number of dopaminergic cells and of the level of dopamine production in each of those cells, the latter of which determines whether TH abundance in each cell is above the threshold for detection. In either case, we expect that an

Table 4

Pattern Matrix Showing Loadings of Each Basal Ganglia egr-1 Measure on Rotated Component 1 or Component 2 in PrincipalComponents Analysis of egr-1 mRNA Levels From All Males

\begin{tabular}{lccc}
\hline $\begin{array}{c}\text { Dorsoventral } \\
\text { site }\end{array}$ & $\begin{array}{c}\text { Rostrocaudal } \\
\text { position }\end{array}$ & Component 1 & Component 2 \\
\hline Dorsal & 1 & 0.0773 & $\mathbf{0 . 7 5 1}$ \\
Ventral & 1 & -0.162 & $\mathbf{0 . 7 3 0}$ \\
Dorsal & 2 & 0.0566 & $\mathbf{0 . 7 5 4}$ \\
Ventral & 2 & 0.0211 & $\mathbf{0 . 8 6 8}$ \\
Dorsal & 3 & $\mathbf{0 . 7 2 9}$ & 0.157 \\
Ventral & 3 & $\mathbf{0 . 6 3 0}$ & 0.16 \\
Dorsal & 4 & $\mathbf{0 . 8 4 9}$ & -0.0467 \\
Ventral & 4 & $\mathbf{0 . 8 9 7}$ & -0.195 \\
\hline
\end{tabular}

Note. Boldface type indicates strong loadings on a factor. increased level of $\mathrm{TH}$ immunoreactivity would correspond to elevated dopamine availability in targets of these PT neurons. The precise time course of this elevated dopamine availability is unknown, however.

Egr-1 mRNA expression typically reflects the amount of depolarization that has occurred in the previous $30 \mathrm{~min}$ and thus is a short-term measure of neural activation (reviewed in Clayton, 2000; Jarvis, 2004). Changes in TH protein abundance likely occur with a longer time course than does egr-1 mRNA induction; thus, we interpret the positive correlation as suggesting that high levels of PT dopamine may cause an increase in pallidal activation rather than the reverse. The lack of correlation between numbers of TH-immunoreactive cells and striatal egr-1 levels may reflect nonlinear or heterogeneous electrophysiological effects of dopaminergic input on striatal neurons. Dopaminergic inputs to the striatum activate multiple receptor subtypes and may modulate voltage-dependent conductances, excitatory synaptic transmission, or inhibitory synaptic transmission (Nicola, Surmeier, \& Malenka,

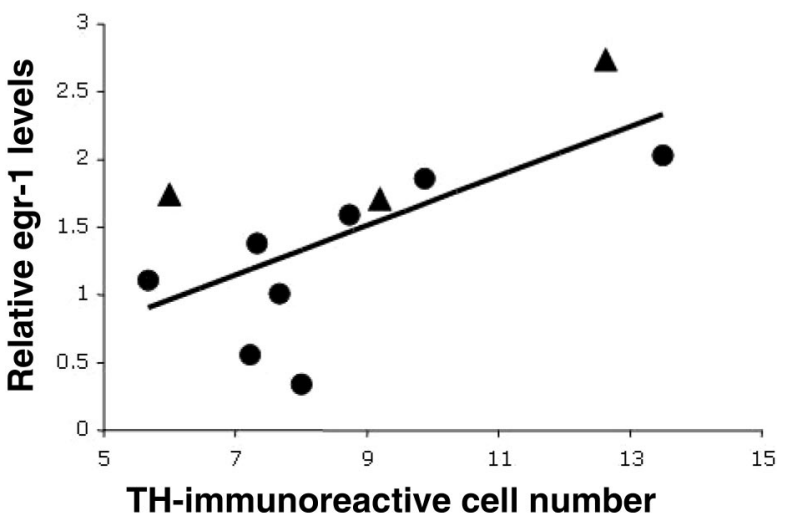

Figure 4. Functional coupling of dopaminergic cells in the posterior tuberculum and neurons in the basal ganglia. The density of THimmunoreactive cell bodies in the posterior tuberculum was correlated with the egr-1 mRNA abundance in the dorsal pallidum. Frogs that heard advertisement calls from conspecific (circles) and heterospecific (triangles) $P$. petersi males appeared to have similar functional coupling. $\mathrm{TH}=$ tyrosine hydroxylase. 
2000). This variation in responses may be reflected more consistently in overall influence on pallidal activation (estimated by pallidal egr- 1 expression) than by depolarization of striatal neurons (estimated by striatal egr- 1 mRNA levels).

Our results have provided additional support to the role for a dopaminergic projection from the PT in regulation of basal ganglia activity in frogs, which is in agreement with previous studies that used lesions, pharmacological manipulations, and anatomical tract tracing. In a manner similar to the basal ganglia circuit in mammals, the dopaminergic cells in the PT may positively influence basal ganglia activation, which could result in modulation of motor output via brainstem motor control areas. The basal ganglia pathways regulate a number of different motor functions, as described above, and thus variation of dopamine level in the PT may be an important determinant of long- or intermediate-term variation in motor output. For example, estradiol levels correlate with the number of TH-immunoreactive cells in the PT of female leopard frogs (Wilczynski, Yang, \& Simmons, 2003). If this increase in dopamine abundance alters activity in the basal ganglia, as described in this article, we propose that a hormonally induced change in PT dopamine signaling may contribute to the variation in likelihood of phonotactic responses to conspecific signals across the reproductive cycle described by Lynch, Rand, Ryan, and Wilczynski (2005). Future experiments that combine natural variation in more stable measures of the state of neural circuits with short-term measures of activation as well as of motor output may reveal how local neurochemical fluctuations may modulate circuit function and thus behavior.

\section{References}

Barbeau, A., Dallaire, L., Buu, N. T., Poirier, J., \& Rucinska, E. (1985). Comparative behavioral, biochemical and pigmentary effects of MPTP, MPP+ and paraquat in Rana pipiens. Life Sciences, 37, 1529-1538.

Barbeau, A., Dallaire, L., Buu, N. T., Veilleux, F., Boyer, H., de Lanney, L. E., et al. (1985). New amphibian models for the study of 1-methyl-4-phenyl-1, 2, 3, 6-tetrahydropyridine (MPTP). Life Sciences, 36, 1125-1134.

Chu, J., \& Wilczynski, W. (2007). Apomorphine effects on frog locomotion behavior. Physiology \& Behavior, 91, 71-76.

Clayton, D. F. (2000). The genomic action potential. Neurobiology of Learning and Memory, 74, 185-216.

Endepols, H., Roden, K., Luksch, H., Dicke, U., \& Walkowiak, W. (2004). Dorsal striatopallidal system in anurans. Journal of Comparative Neurology, 468, 299-310.

Endepols, H., Schul, J., Gerhardt, H. C., \& Walkowiak, W. (2004). 6-hydroxydopamine lesions in anuran amphibians: A new model system for Parkinson's disease? Journal of Neurobiology, 60, 395-410.

Finkenstädt, T. (1989). Visual associative learning: Searching for behaviorally relevant brain structures in toads. In J.-P. Ewert \& M. A. Arbib (Eds.), Visuomotor coordination (pp. 799-832). New York: Plenum.

Glagow, M., \& Ewert, J.-P. (1996). Apomorphine-induced suppression of prey oriented turning in toads is correlated with activity changes in pretectum and tectum: ${ }^{14} \mathrm{C}-2 \mathrm{DG}$ studies and single cell recordings. Neuroscience Letters, 220, 215-218.

Glagow, M., \& Ewert, J.-P. (1997). Dopaminergic modulation of visual responses in toads: I. Apomorphine-induced effects on visually directed appetitive and consummatory prey-catching behavior. Journal of Comparative Physiology A, 180, 1-9.

Glagow, M., \& Ewert, J.-P. (1999). Apomorphine alters prey-catching patterns in the common toad: Behavioral experiments and ${ }^{14} \mathrm{C}-2$ deoxyglucose brain mapping studies. Brain, Behavior and Evolution, 54, 223-242.
González, A., \& Smeets, W. J. (1991). A comparative analysis of dopamine-immunoreactivity in the brain of two amphibians, the anuran Rana ridibunda and the urodele Pleurodeles waltlii. Journal of Comparative Neurology, 303, 457-477.

Hoke, K. L., Burmeister, S. S., Fernald, R. D., Rand, A. S., Ryan, M. J., \& Wilczynski, W. (2004). Functional mapping of the auditory midbrain during mate call reception. Journal of Neuroscience, 24, 11264-11272.

Hoke, K. L., Ryan, M. J., \& Wilczynski, W. (2005). Social cues shift functional connectivity in the hypothalamus. Proceedings of the $\mathrm{Na}$ tional Academy of Sciences, USA, 102, 10712-10717.

Hoke, K. L., Ryan, M. J., \& Wilczynski, W. (2007). Integration of sensory and motor processing underlying social behaviour in túngara frogs. Proceedings of the Royal Society B, 274, 641-649.

Jarvis, E. D. (2004). Brains and birdsong. In P. Marler \& H. Slabberkoorn (Eds.), Nature's music: The science of birdsong (pp. 239-275). New York: Elsevier-Academic Press.

Lynch, K. S., Rand, A. S., Ryan, M. J., \& Wilczynski, W. (2005). Plasticity in female mate choice associated with changing reproductive states. Animal Behaviour, 69, 689-699.

Marín, O., González, A., \& Smeets, W. J. (1997a). Anatomical substrate of amphibian basal ganglia involvement in visuomotor behaviour. European Journal of Neuroscience, 9, 2100-2109.

Marín, O., González, A., \& Smeets, W. J. (1997b). Basal ganglia organization in amphibians: Afferent connections to the striatum and the nucleus accumbens. Journal of Comparative Neurology, 378, 16-49.

Marín, O., González, A., \& Smeets, W. J. (1997c). Basal ganglia organization in amphibians: Efferent connections of the striatum and the nucleus accumbens. Journal of Comparative Neurology, 380, 23-50.

Marín, O., Smeets, W. J., \& González, A. (1997). Basal ganglia organization in amphibians: Catecholaminergic innervation of the striatum and nucleus accumbens. Journal of Comparative Neurology, 378, 50-69.

Marín, O., Smeets, W. J., \& González, A. (1998). Evolution of the basal ganglia in tetrapods: A new perspective based on recent studies in amphibians. Trends in Neurosciences, 21, 487-494.

Mühlenbrock-Lenter, S., Endepols, H., Roth, G., \& Walkowiak, W. (2005). Immunohistological characterization of striatal and amygdalar structures in the telencephalon of the fire-bellied toad Bombina orientalis. Neuroscience, 134, 705-719.

Nicola, S. M., Surmeier, D. J., \& Malenka, R. C. (2000). Dopaminergic modulation of neuronal excitability in the striatum and nucleus accumbens. Annual Review of Neuroscience, 23, 185-215.

Patton, P., \& Grobstein, P. (1998). The effects of telencephalic lesions on visually mediated prey orienting behavior in the leopard frog (Rana pipiens): II. The effects of limited lesions to the telencephalon. Brain, Behavior and Evolution, 51, 144-161.

Smeets, W. J., \& González, A. (2000). Catecholamine systems in the brain of vertebrates: New perspectives through a comparative approach. Brain Research Reviews, 33, 308-379.

Walkowiak, W., Berlinger, M., Schul, J., \& Gerhardt, H. C. (1999). Significance of forebrain structures in acoustically guided behavior in anurans. European Journal of Morphology, 37, 177-181.

Wilczynski, W., \& Northcutt, R. G. (1983a). Connections of the bullfrog striatum: Afferent organization. Journal of Comparative Neurology, 214, 321-332.

Wilczynski, W., \& Northcutt, R. G. (1983b). Connections of the bullfrog striatum: Efferent projections. Journal of Comparative Neurology, 214, 333-343.

Wilczynski, W., Yang, E.-J., \& Simmons, D. (2003). Sex differences and hormone influences on tyrosine hydroxylase cells in the leopard frog. Journal of Neurobiology, 56, 54-65.

Received April 18, 2007

Revision received September 12, 2007 Accepted September 18, 2007 